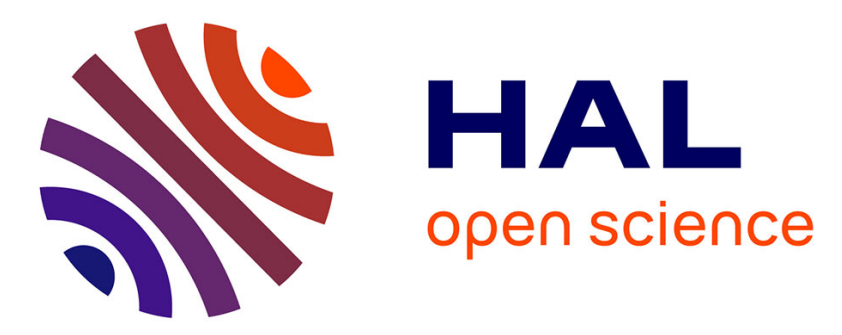

\title{
Quantitative treatment of multiple flux quantum transition by comparing an analog computer simulation with a simple analytical approach
}

G. Voss, A. Hausen

\section{- To cite this version:}

G. Voss, A. Hausen. Quantitative treatment of multiple flux quantum transition by comparing an analog computer simulation with a simple analytical approach. Revue de Physique Appliquée, 1982, 17 (5), pp.295-300. 10.1051/rphysap:01982001705029500 . jpa-00244999

HAL Id: jpa-00244999

https://hal.science/jpa-00244999

Submitted on 1 Jan 1982

HAL is a multi-disciplinary open access archive for the deposit and dissemination of scientific research documents, whether they are published or not. The documents may come from teaching and research institutions in France or abroad, or from public or private research centers.
L'archive ouverte pluridisciplinaire HAL, est destinée au dépôt et à la diffusion de documents scientifiques de niveau recherche, publiés ou non, émanant des établissements d'enseignement et de recherche français ou étrangers, des laboratoires publics ou privés. 


\title{
Quantitative treatment of multiple flux quantum transition by comparing an analog computer simulation with a simple analytical approach
}

\author{
G. Voss and A. Hausen $\left({ }^{*}\right)$ \\ II. Physikalisches Institut der Universität zu Köln, D-5000 Köln 41, R.F.A.
}

(Reçu le 28 septembre 1981, révisé le 10 février 1982, accepté le 15 février 1982)

\begin{abstract}
Résumé. - Il est décrit un moyen d'ordinateur analogique pour le traitement quantitatif de la transition multiple des quanta du flux dans une bague superconductible faiblement couplée pour toute relation courant-phase (CPR). Les résultats obtenus pour les CPR sinusoïdales ou sinusoïdal modifiées sont comparés à ceux d'un mode d'exécution analytique simple.

En raison de la concordance excellente, une application technique du phénomène de la pénétration du flux multiple pourrait être contrôlée par les données du mode d'exécution analytique.

Abstract. - An analog computer device is described for a quantitative treatment of multiple flux quantum transition into a weakly connected superconducting (sc) loop for any current phase relation (CPR).

For sinusoidal and modified sinusoidal CPR the results of the analog computer calculations are compared with the results of a simple analytical approach.

Since there is an excellent agreement, a technical application of the multiple flux penetration phenomenon might be controlled by the data of the analytical approach
\end{abstract}

1. Introduction. - Recently we reported on experiments concerning multiple flux quantum transitions in superconducting (sc) loops containing a $\mathrm{Nb}-\mathrm{Nb}$ point contact as weak link [1].

The experimental results were analysed and discussed using the phenomenological Resistively Shunted Junction (RSJ)-model [2]. Moreover, it was shown, that the correlation between flux quantum transition and $I-U$-characteristic can be described quantitatively by the RSJ-model in case of sinusoidal or modified sinusoidal current phase relations. Within the frame of this model the dynamics of flux penetration are governed by the differential equation

$$
\phi=\phi_{\mathrm{x}}-L\left[C \frac{\mathrm{d}^{2} \phi}{\mathrm{d} t^{2}}+\frac{1}{R} \frac{\mathrm{d} \phi}{\mathrm{d} t}+I_{\mathrm{c}} f(\phi)\right] .
$$

$\phi$ is the flux actually threading the loop, $\phi_{x}$ is the flux externally applied to the loop, $L$ is the inductance of the loop, $C$ is the capacitance, $R$ the resistance, $I_{\mathrm{c}}$ the critical current and $I_{\mathrm{c}} f(\phi)$ the current phase relation (CPR) of the weak link.

$\left(^{*}\right)$ Present address : McKinsey \& Co., Düsseldorf, West Germany.
In this paper we will examine the solution $\phi(t)$ by analog computer simulation as well as by an analytical approximation which was already discussed in [1]

For small values of $\Gamma=L I_{\mathrm{c}} / \phi_{0}(\Gamma<10)\left(\phi_{0}=2.068 \times\right.$ $10^{-15} \mathrm{Vs}$ is the flux quantum) analog computer calculations (described in section 2) have been done in case of sinusoidal and triangular CPR using a new and simple technique to simulate the nonlinear part in (1). Results are presented in section 2.2.

In sections 3 and 4 the analytical approximation originally introduced in [1] for large values of $\Gamma$ $(\Gamma \gg 10)$ - is applied to the case $\Gamma<10$ treated by analog computer simulation in section 2 .

In section 4 we will show that the results from analog computer simulation and analytical approximation are in excellent agreement, that means the analytical approximation is well applicable in the regime $\Gamma<10$ too.

2. Analog computer simulation. - 2.1 EXPERIMENTAL ARRANGEMENT. - Using dimensionless variables

$$
\varphi_{(\mathrm{x})}=\frac{\phi_{(\mathrm{x})}}{\phi_{0}}, \quad t_{1}=\frac{1}{\sqrt{L C}} t
$$


and parameters

$$
\Gamma=\frac{L I_{\mathrm{c}}}{\phi_{\mathrm{o}}}, \quad \beta=\frac{\sqrt{L C}}{R C}
$$

one obtains an appropriate form for the analog computer simulation of equation (1) :

$$
\begin{gathered}
\varphi^{\prime \prime}+\beta \varphi^{\prime}+\varphi+\Gamma f(\varphi)=\varphi_{\mathrm{x}}, \\
\varphi(0)=0, \quad \varphi^{\prime}(0)=0
\end{gathered}
$$

denotes the derivative with respect to $t_{1}$.

In case of sinusoidal CPR $[f(\varphi)=\sin (2 \pi \varphi)]$ the properties of this differential equation were studied by several authors using digital [3]-[8] or analog [9] computer techniques. Moreover, in [4] and [7]-[9] the effect of the $\cos (2 \pi \varphi)$-term was taken into consideration.

The analog computer circuit used in this work consists of an arrangement of operational amplifier-elements (simulation of the linear parts in $(1 a)$ ) and a section discussed below for the simulation of the nonlinear part in (1a), i.e. the CPR. Evaluating the blockdiagram of the circuit (see Fig. 1) it's easy to show that

$$
\begin{array}{r}
-U^{\prime}=\int_{0}^{T_{1}} \mathrm{~d} T_{1}\left[U_{\mathrm{x}}-10 p_{1} U^{\prime}-U\right. \\
\left.-100 p_{2} A f\left(\frac{. U}{U_{0}}\right)\right] \\
U^{\prime \prime}+10 p_{1} U^{\prime}+U+100 p_{2} A f\left(\frac{U}{U_{0}}\right)=U_{\mathrm{x}}
\end{array}
$$

denotes the derivative with respect to the dimensionless time $T_{1}=1 \mathrm{~Hz} . T$. Comparing the equations $(1 a)$ and (2) one yields the relations :

$$
\begin{aligned}
\phi_{(\mathbf{x})} & =\frac{\phi_{(\mathbf{x})}}{\phi_{0}} \triangleq \frac{U_{(\mathbf{x})}}{U_{0}} \\
t_{1} & =\frac{1}{\sqrt{L C}} t \triangleq T_{1}=1 \mathrm{~Hz} . T \\
\Gamma & =\frac{L I_{\mathrm{c}}}{\phi_{0}} \triangleq 100 p_{2} A \\
\beta & =\frac{\sqrt{L C}}{R C} \cong 10 p_{1}
\end{aligned}
$$

$T$ : Equivalent time, $t:$ Real time.

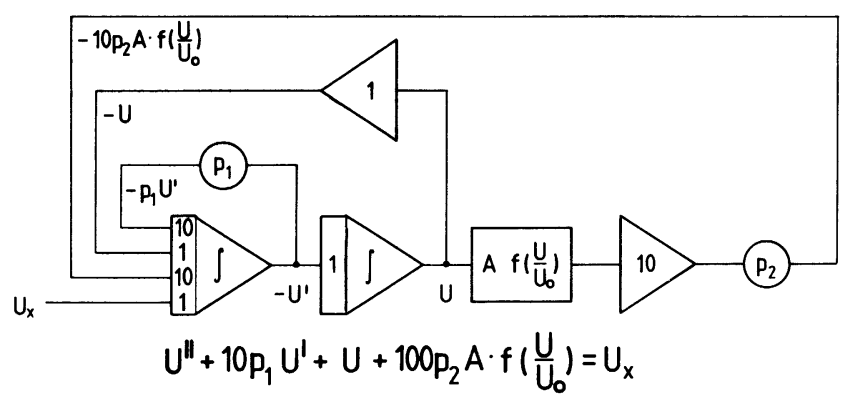

Fig. 1. - Block-diagram of the analog computer circuit; for further explanation of the section $A f\left(U / U_{0}\right)$; see figure 2 !
For the simulation of the nonlinear part in (1a) we used a function generator in combination with a sampling scope. In principle the signal $U_{\mathrm{a}}=A f\left(\frac{U_{\mathrm{e}}}{U_{0}}\right)$ is generated in the following way : The periodic output signal of the function generator

$$
U_{\mathrm{G}}(t) \propto f(t)
$$

whose shape corresponds to the actual CPR $f(\varphi)$, serves as the vertical deflection for the sampling scope. At an appropriate, fixed time deflection a standing pattern is displayed which can be scanned by an external voltage $U_{\mathrm{e}} . U_{\mathrm{e}}$ determines the abscissa $t$ in (3). The output $U_{\mathrm{a}}$ is proportional to the corresponding ordinate $U_{\mathrm{G}}(t)$, that means, there is a conversion $U_{\mathrm{G}}\left(t\right.$ ) into $U_{a}\left(U_{\mathrm{e}}\right)$ (Fig. 2b). If $U_{\mathrm{e}}$ is time dependent (Fig. 2a) : $U_{\mathrm{e}}=U_{\mathrm{e}}(T), U_{\mathrm{a}}\left(U_{\mathrm{e}}\right)$ is transformed into $U_{\mathrm{a}}(T)=U_{\mathrm{a}}\left[U_{\mathrm{e}}(T)\right]$ (Fig. $\left.2 c\right)$, which can be fed back into the analog computer part.

b)

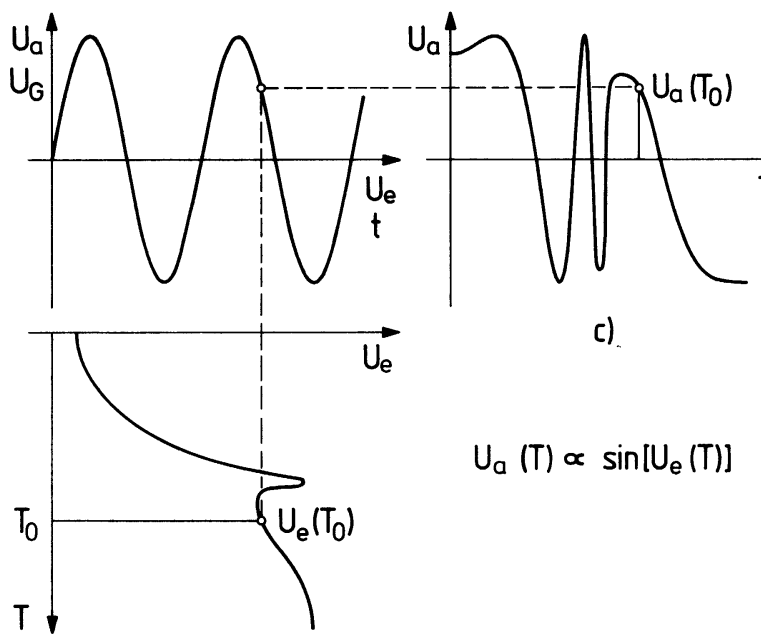

Fig. 2. - Principle for generating the signal $U_{\mathrm{a}}(T)=$ $A f\left[U_{\mathrm{e}}(T)\right]$; here $f(\varphi)=\sin 2 \pi \varphi$. By means of the $U_{\mathrm{a}}-U_{\mathrm{e}}-$ characteristic $(b)$ the input signal $U_{e}(T)(a)$ is transformed into the output signal $U_{\mathrm{a}}(T)(c)$.

This purely analog calculation is only limited by the number of sampling points which are available for displaying the periodic signal $U_{G}(t)$. In practice up to 50 periods can be recorded.

The main advantage of the method just described is that one can simulate all types of CPR's as long as a corresponding waveform can be generated by the function generator.

2.2 RESUlts. - In figure 3 the time dependence of $\varphi$ and $\mathrm{d} \varphi / \mathrm{d} t_{1}$ during a multiple flux quantum transition is shown in case of the sinusoidal $(a)$ as well as in case of a triangular (b) CPR for identical $\Gamma=8$ and $\beta=2.6$. The graph of the triangular CPR characterized by an excess current $I_{2}=\frac{1}{2} I_{\mathrm{c}}$ is shown in [1], figure $6 a$. 


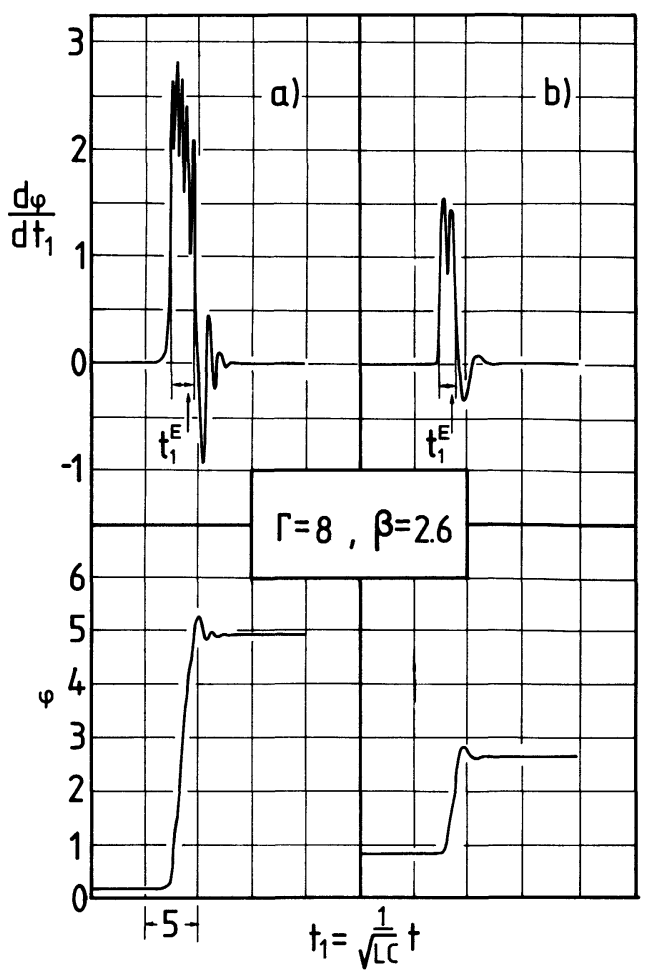

Fig. 3. $-\varphi\left(t_{1}\right), \varphi^{\prime}\left(t_{1}\right)$-curves obtained by analog computer simulation of the differential equation $(1 a)$ for the parameters $\Gamma=8, \beta=2.6:(a)$ sinusoidal CPR; $(b)$ triangular CPR.

When the external flux $\varphi_{\mathrm{x}}$ exceeds the critical value $\varphi_{\mathrm{xc}} \simeq \frac{L I_{\mathrm{c}}}{\varphi_{0}}=\Gamma$, a flux quantum transition is triggered, and $\varphi$ varies rapidly in time. $\varphi_{\mathrm{x}}$ is linearly increased in time; but during the transition it varies so slowly compared to the time variation of $\varphi$ that it can be assumed to be constant.

The number of the oscillations on top of the voltagepulses $\left(U \propto \frac{\mathrm{d} \varphi}{\mathrm{d} t_{1}}\right)$ is determined by the number of the flux quanta $\varphi_{\mathrm{E}}$ penetrating into the loop. In case of the sinusoidal CPR (Fig. $3 a$ ) the flux $\varphi$ changes by an amount of five, in case of the triangular CPR (Fig. $3 b$ ) by an amount of two flux quanta.

The penetration time for the flux $t_{1}^{\mathrm{E}}=\frac{1}{\sqrt{L C}} t_{\mathrm{E}}$ has been evaluated from the width of the $\frac{\mathrm{d} \varphi}{\mathrm{d} t_{1}}$-pulse at $\left(\frac{\mathrm{d} \varphi}{\mathrm{d} t_{1}}\right)_{\max } / 2$. Moreover, the mean penetration frequency for the flux $v_{M}$ can be obtained according to the definition

$$
\sqrt{L C} v_{\mathrm{M}} \equiv \frac{\varphi_{\mathrm{E}}}{t_{1}^{\mathrm{E}}}
$$

Typical values for $t_{\mathrm{E}}$ and $v_{\mathrm{M}}$ range in the Picosecond - and the Gigahertz - regime respectively, e.g. supposing $L=1 \mathrm{nH}, C=0.3 \mathrm{pF}, R=22 \Omega$ and
$I_{\mathrm{c}}=16 \mu \mathrm{A}$ (this is just the case $\Gamma=8, \beta=2.6$ ) we obtain

$$
\begin{aligned}
& t_{\mathrm{E}} \equiv \sqrt{L C} t_{1}^{\mathrm{E}} \simeq 42 \mathrm{ps} \\
& v_{\mathrm{M}} \equiv \frac{\varphi_{\mathrm{E}}}{t_{\mathrm{E}}} \simeq 120 \mathrm{GHz}
\end{aligned}
$$

with $\varphi_{\mathrm{E}}=5, t_{1}^{\mathrm{E}}=2.4$ taken from figure $3 a$.

A survey of the results of the analog computer experiments is given in figure 4 (number of penetrated flux quanta $\varphi_{\mathrm{E}}$ per transition $v s$. damping parameter $\beta$ ) and figure 5 (normalized mean penetration frequency for the flux $\sqrt{L C} v_{\mathrm{M}} v$ s. $\beta$ ).

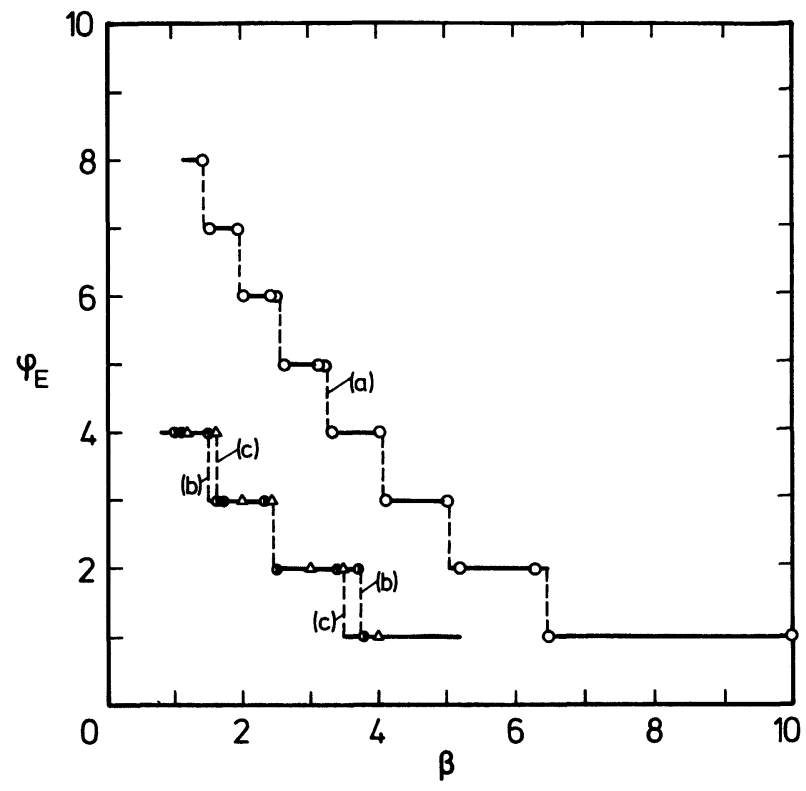

Fig. 4. - Number of penetrated flux quanta $\varphi_{\mathrm{E}}$ per transition vs. $\beta$ in case of : $(a)$ sinusoidal CPR; $(b)$ modified sinusoidal CPR; (c) triangular CPR. The graphs of the modified sinusoidal and the triangular CPR are shown in [1], figure 6 .

O sinusoidal CPR; $O$ modified sinusoidal CPR ; $\triangle$ triangular CPR.

Obviously $\varphi_{\mathrm{E}}$ and $\sqrt{L C} v_{\mathrm{M}}$ change discontinuously at identical, discrete values of $\beta$.

In case of the sinusoidal CPR $(a) L I_{\mathrm{c}} / \phi_{0}$ determines the maximum number of flux quanta to penetrate, which in case of modified sinusoidal $(b)$ and triangular (c) CPR is diminuished by $L I_{2} / \phi_{0}$.

For the triangular CPR we obtain nearly the same results as in case of modified sinusoidal CPR characterized by the same excess current (see [1], Fig. 6).

3. Analytical approximation. - In [1] we have discussed an analytical approximation for the nonlinear differential equation (1). This approximation is mainly based on the assumptions :

- that during a flux quantum transition $\varphi_{\mathrm{x}}$ varies slowly compared to the time variation of $\varphi$; 


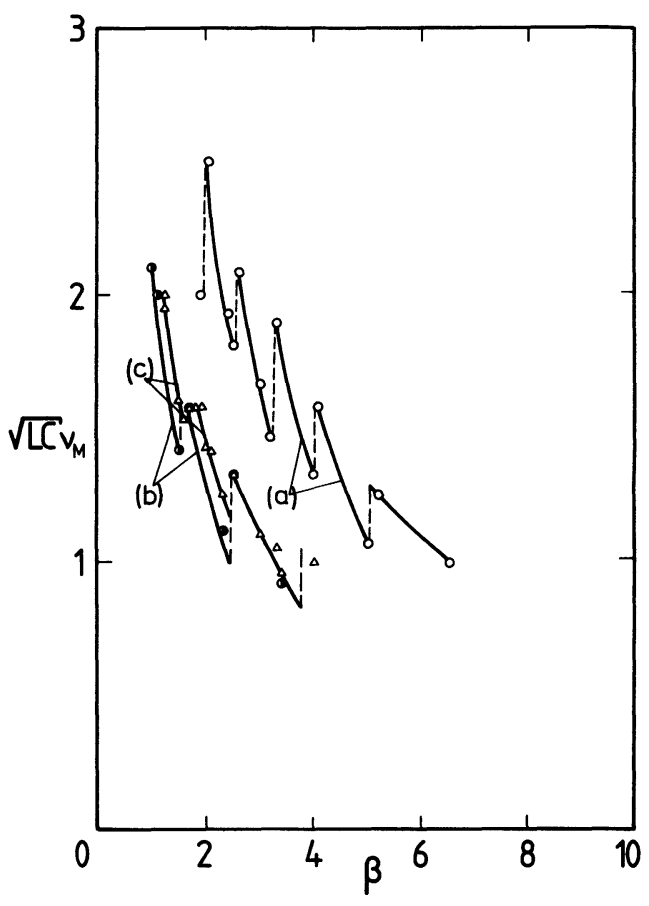

Fig. 5. - Normalized mean penetration frequency for the flux $\sqrt{L C} v_{\mathrm{M}}$ vs. $\beta$ in case of :(a) sinusoidal CPR;(b) modified sinusoidal CPR; (c) triangular CPR.

- and that the rapidly oscillating supercurrent $I_{\mathrm{s}}(t)=I_{\mathrm{c}} f[\varphi(t)]$ can be replaced by its time average value, which is just the excess current $I_{2}$.

Although the linear differential equations, which result from the approximation can be solved easily (provided $\beta>2$ ) [see [1], equations (20), (21) !], it is necessary to find the time $t_{\mathrm{E}}$ at which flux penetration should stop. As discussed in [1] $t_{\mathrm{F}}$ is determined by the hysteresis-parameter $\alpha_{1}^{+}$and

$$
\Gamma^{+}=L I_{\mathrm{c}}^{+} / \phi_{0} \quad\left(I_{\mathrm{c}}^{+}=I_{\mathrm{c}}-I_{2}\right),
$$

which again depend on the actual CPR. So the CPR is involved in our calculations via the termination condition for flux penetration.

Since in case of sinusoidal and modified sinusoidal CPR the dependence of $\alpha_{1}^{+}$on $\beta$ is known :

$$
\begin{aligned}
\varphi_{\mathrm{E}} \equiv \varphi_{\mathrm{E}}(x)=\Gamma^{+}\left(1-\alpha_{1}^{+}(x)\right) \\
\alpha_{1}^{+}(x)=\frac{x}{2(4-\pi)}[-(\pi-2) x+ \\
\quad+\sqrt{\left.(\pi-2)^{2} x^{2}+8(4-\pi)\right]} \\
x=\sqrt{\frac{I_{\mathrm{c}}}{I_{\mathrm{c}}^{+}}} \frac{\beta}{\beta_{\mathrm{c}}}, \quad \beta_{\mathrm{c}}=\sqrt{\frac{2 \pi}{4-\pi}} \sqrt{\Gamma}=2.71 \sqrt{\Gamma}
\end{aligned}
$$

the voltage pulse $\mathrm{d} \varphi / \mathrm{d} t_{1}$ and $\mathrm{d} \phi / \mathrm{d} t$ respectively can be easily calculated.

Figure 6 shows the plots of equation (6) for the sinusoidal CPR $\left(\Gamma=\Gamma^{+}=8, I_{2} / I_{\mathrm{c}}=0 \rightarrow\right.$ curve $\left.(d)\right)$ and for the modified sinusoidal CPR $\left(\Gamma=8, \Gamma^{+}=4\right.$, $\left.I_{2} / I_{\mathrm{c}}=0.5 \rightarrow \operatorname{curve}(e)\right)$ in comparison with the

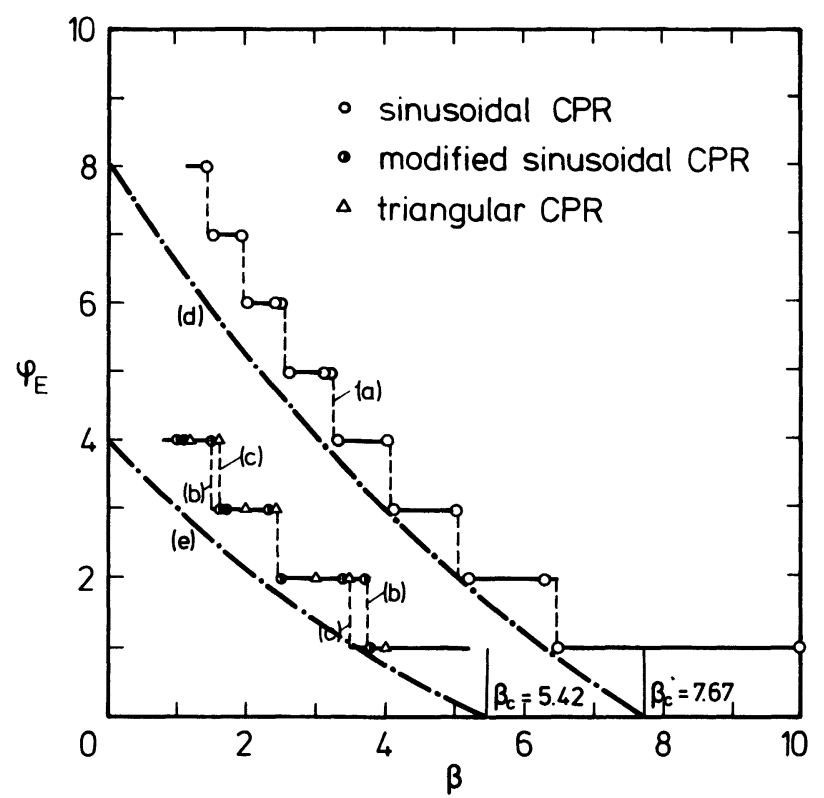

Fig. 6. - Dependence of penetrating flux $\varphi_{\mathrm{E}}$ on the parameter $\beta\left(\beta_{\mathrm{c}}=2.71 \sqrt{\Gamma}\right)$. Plots of equation $(6):(d)$ sinusoidal CPR $\left(\Gamma=\Gamma^{+}=8, I_{2} / I_{\mathrm{c}}=0\right) ;(e)$ modified sinusoidal CPR $\left(\Gamma=8, \Gamma^{+}=4, I_{2} / I_{\mathrm{c}}=0.5\right)$, in comparison with the results obtained by analog computer simulation $(a)-(c)$ [see Fig. 4 !].

results obtained by analog computer simulation (Fig. 4).

Although equation (6) was originally stated in [1] for large values of $\Gamma(\Gamma>10)$, there is an excellent agreement for $\Gamma=\Gamma^{+}=8$ and $\Gamma^{+}=4$ respectively between the discontinuous curves $(a)$ - $(c)$ (analog computer simulation) and the continuous curves $(d),(e)$ (analytical calculation).

For small damping $(\beta \lesssim 1)$ the analog computer simulations became unstable and the prediction of equation (6) : $\varphi_{\mathrm{E}} \rightarrow \Gamma^{+}$for $\beta \rightarrow 0$ couldn't be verified. However, the regime $0<\beta<2$ has been extensively studied by numerical calculations in [6] and it has been shown that the behaviour of the system is erratic for $\beta \lesssim 1.6$.

In the following we are treating only the case $\beta>2$. Then we get for the voltage pulse $\mathrm{d} \phi / \mathrm{d} t(\beta \gg 2)$ :

$$
\frac{\mathrm{d} \phi}{\mathrm{d} t}=\left\{\begin{array}{c}
R I_{\mathrm{c}}^{+}\left[\exp \left(-\frac{t}{\tau^{*}}\right)-\exp \left(-\frac{t}{\tau}\right)\right] \\
R I_{\mathrm{c}}^{+} \alpha_{1}^{+} \exp \left(-\frac{t-t_{\mathrm{E}}}{\tau}\right) ; \quad 0 \leqslant t \leqslant t_{\mathrm{E}} \\
\left(\tau^{*}=L / R, \quad \tau=R C\right) .
\end{array}\right.
$$

Moreover, assuming $t_{\mathrm{E}} \gg \tau$ we obtain an analytical expression for the normalized mean penetration frequency :

$$
\sqrt{L C} v_{\mathrm{M}} \equiv \frac{\varphi_{\mathrm{E}}}{t_{1}^{\mathrm{E}}}=-\frac{\Gamma^{+}}{\beta} \cdot \frac{1-\alpha_{1}^{+}(\beta)}{\ln \alpha_{1}^{+}(\beta)} .
$$


In section 4 these results for $\mathrm{d} \phi / \mathrm{d} t$ and $\sqrt{L C} v_{\mathrm{M}}$ will be discussed in comparison with the corresponding results of the analog computer simulations.

4. Discussion. - Since $t_{\mathrm{E}}$ and $v_{\mathrm{M}}$ are of the order of Picoseconds and Gigahertz respectively, and since the area under the voltage pulse is equal to the penetrated flux (typically some $\phi_{0}$ ), the pulse structure cannot be subject to individual real-time measurements.

However, by analog computer simulation the dynamics of flux penetration can be studied in an equivalent time domain as presented in section 2 .

For sufficiently large $\Gamma\left(\Gamma \simeq 10^{4}\right)$ the comparison of the analytical approximation with the individually measured pulses leads to an excellent agreement [1]. For small values of $\Gamma(\Gamma<10)$ this comparison must be done with the analog computer results and is presented in figures 7 and 8.

In figure 7 analytically calculated $\mathrm{d} \varphi / \mathrm{d} t_{1}$-pulses are shown in comparison with the corresponding $\mathrm{d} \varphi / \mathrm{d} t_{1^{-}}$ pulses obtained by analog computer simulation (Fig. 3).

Apparently the calculated pulse cannot exhibit the quantum nature of the discontinuous flux penetration process and averages the oscillations on top of the corresponding pulse obtained by analog computer simulation. However, there is an excellent agreement concerning the overall pulse shape. Note for instance that the leading and trailing edges of the corresponding pulses are nearly indistinguishable.

Consequently the areas under the corresponding pulses are nearly the same (the deviations are less than one flux quantum).

The dependence of the normalized mean penetration frequency for the flux $\sqrt{L C} v_{M}$ on the parameter $\beta$ is shown in figure 8. The results from the analytical calculation [curves $(e),(g)$ ] agree very well with the results obtained by analog computer simulation [curves $(a)-(c)$ ] for $2<\beta<\beta_{\mathrm{c}}$.

Whereas the curves $(e),(g)$ tend to zero in the limit $\beta \rightarrow \beta_{\mathrm{c}}\left({ }^{1}\right)$, the curves $(d),(f)$ which we obtained from equation (8) tend to the finite value $\Gamma^{+} / \beta_{\mathrm{c}}$ for $\beta \rightarrow \beta_{\mathrm{c}}$.

Moreover, we see that equation (8) gives an excellent estimation for $\sqrt{L C} v_{\mathrm{M}}$ in the regime $\Gamma<10$ too.

The investigations do not lead to a better understanding of the physics of weakly connected sc loops but give a tool for quantitative evaluation of the multiple flux penetration phenomenon.

Several experiments and calculations have been made in order to understand the dynamics of flux quantum transitions. We have tried to arrange these works in table I with respect to the CPR and the regime of $\beta$.

( $\left.{ }^{1}\right)$ This tendency is unphysical, since even at high damping $\left(x=\beta / \beta_{c}>1\right)$ one flux quantum will penetrate into a weakly connected sc loop in a finite time.

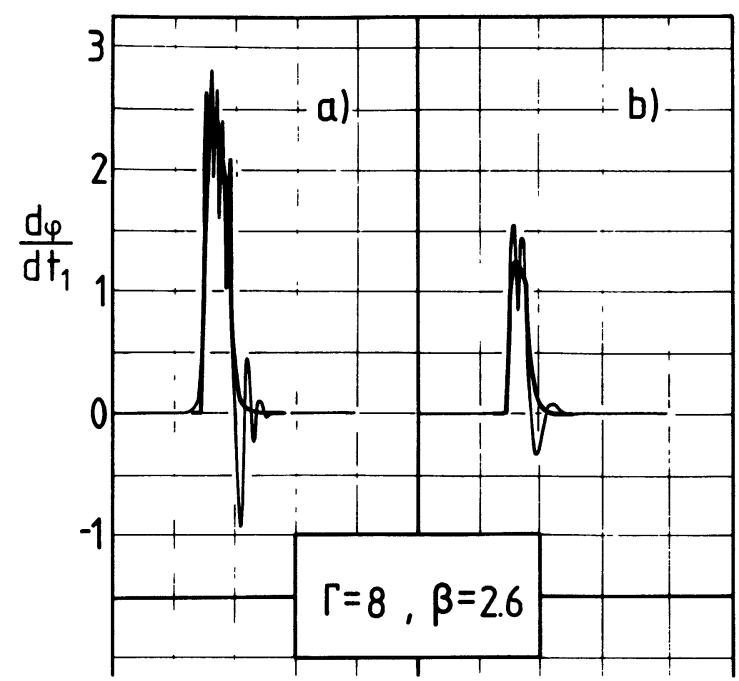

Fig. 7. - Comparison of the $\varphi^{\prime}\left(t_{1}\right)$-curves obtained by

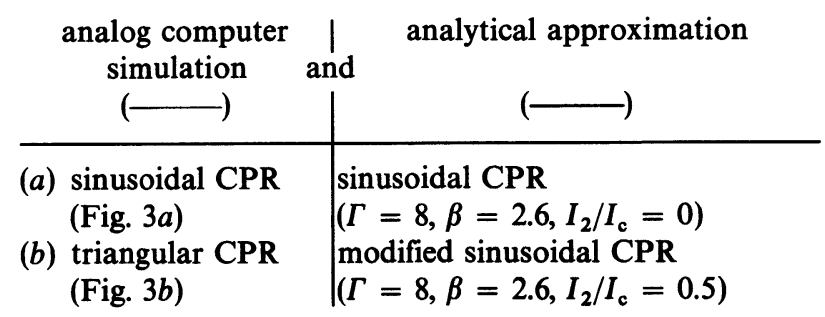

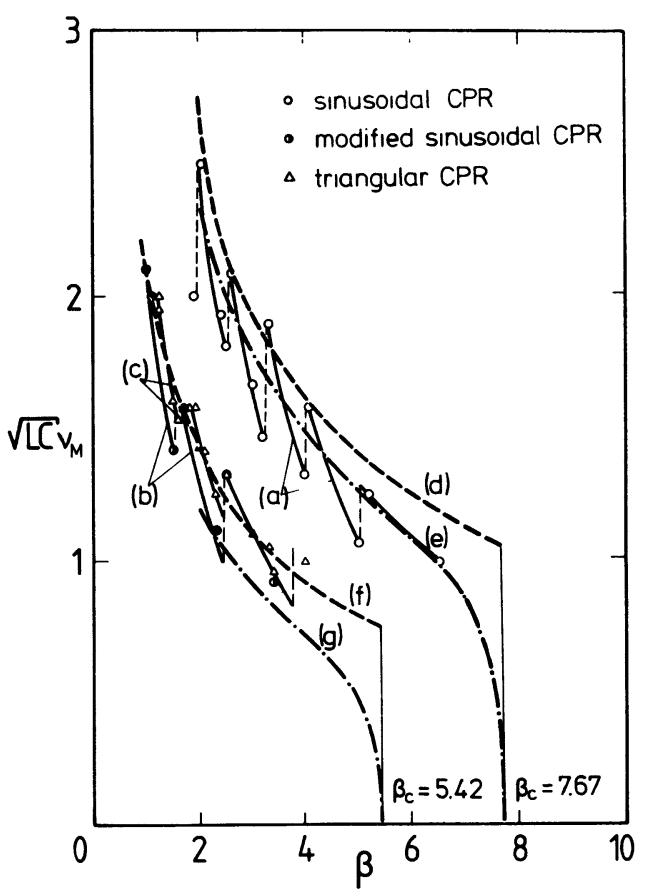

Fig. 8. - Dependence of the normalized mean penetration frequency for the flux $\sqrt{L C} v_{\mathrm{M}}$ on the parameter $\beta$ $\left(\beta_{\mathrm{c}}=2.71 \sqrt{\Gamma}\right)$. Analytical calculation : $(e)$ sinusoidal CPR; $(g)$ modified sinusoidal CPR. Plots of equation (8) : $(d)$ sinusoidal CPR; $(f)$ modified sinusoidal CPR, in comparison with the results obtained by analog computer simulation (a)-(c) [see Fig. 5 !].

For $0<\beta<2$ only calculations have been made as this case is not yet relevant for applications. 
Table I.

\begin{tabular}{|c|c|c|c|c|}
\hline & & $f(\varphi)$ & & \\
\hline$\beta$ & $\sin (2 \pi \varphi)$ & $\begin{array}{l}\sin (2 \pi \varphi) \\
+\cos (2 \pi \varphi) \text {-term }\end{array}$ & $\pm a+b \sin \left[2 \pi\left(\varphi \mp \varphi_{0}\right)\right]$ & triangular \\
\hline $\begin{array}{l}\text { experimental results } \\
\text { are discussed in terms } \\
\text { of } f(\varphi)\end{array}$ & [3] & [4] & [1] & [1] \\
\hline$\beta>2$ & $\begin{array}{l}\text { analog } \\
{[9],[*]} \\
\text { analytical approximation } \\
\text { [1], [*] } \\
\text { digital } \\
{[3],[5],[7],[8]}\end{array}$ & $\begin{array}{l}\text { analog } \\
{[9]} \\
\text { digital } \\
{[4],[7],[8]}\end{array}$ & $\begin{array}{l}\text { analog } \\
{[*]} \\
\text { analytical approximation } \\
{[1],[*]}\end{array}$ & $\begin{array}{l}\text { analog } \\
{[*]}\end{array}$ \\
\hline $\begin{array}{c}\text { calculations only } \\
\qquad \beta<2 \\
\end{array}$ & $\begin{array}{l}\text { digital } \\
{[6]}\end{array}$ & $\begin{array}{l}\text { digital } \\
{[8]} \\
{[*] \text { : this work }}\end{array}$ & & \\
\hline
\end{tabular}

Deviations from the pure sinusoidal behaviour are discussed in terms of the $\cos (2 \pi \varphi)$-term, although there is a big quantitative discrepancy [4]. Improved calculations have been presented in $[7,8]$, but there has been no essential progress in solving the discrepancy.

The experimental results, however, can easily be understood, if one assumes a CPR with a non-vanishing excess current as discussed in [1].

In order to check the sign of the $\cos (2 \pi \varphi)$-term one has to be sure that the CPR is pure sinusoidal. In general this is not the case.

One application of multiple flux penetration could be a fluxmeter that determines the flux by direct counting the number of the voltage pulses. In case of the well known SQUID based on the single flux quantum transition voltage pulses cannot be detected directly but only with high electronical efforts which cause a time constant of $1 \mu$ s per flux quantum. However, there are cases where the sensitivity and accuracy of the SQUID is not needed but a higher time resolution is useful.

It would be desirable to build a device which can be switched from the one flux quantum regime to the multiple flux quantum regime by changing the parameters $R$ and $C$ externally.

\section{References}

[1] Voss, G., Hausen, A., Revue Phys. Appl. 15 (1980) 323.

[2] Stewart, W. C., Appl. Phys. Lett. 12 (1968) 277. McCumber, D. E., J. Appl. Phys. 39 (1968) 3113.

[3] Smith, H. J. T., Blackburn, J. A., Phys. Rev. B 12 (1975) 940.

[4] Blackburn, J. A., Smith, H. J. T., Keith, V., Phys. Rev. B 15 (1977) 4211.

[5] Dempsey, D. G., Levinsen, M. T., Ulrich, B. T., IEEE Trans. Mag. MAG-11 (1975) 811.
[6] Wang, T. C., Gayley,_R. I., Phys. Rev. B 15 (1977) 3401.

[7] Gayley, R. I., Wang, T. C., Phys. Rev: B 16 (1977) 3270.

[8] Peterson, R. L., Gayley, R. I., Phys. Rev. B 18 (1978) 1198.

[9] ERnÉ, S. N., LUTHER, H., PTB Berlin, private communication. 